\title{
Nuove tecniche di transgenesi e imaging: neuro e nefro-applicazioni. Parte 1
}

\author{
Duccio Lombardi ${ }^{1}$, Tommaso Alterini ${ }^{2}$
}

${ }^{1}$ Dottorando in Area del Farmaco e Trattamenti Innovativi, Dipartimento di Medicina Sperimentale e Clinica, Centro d'Eccellenza DENOTHE, Università degli Studi di Firenze, Firenze

${ }^{2}$ Laureando magistrale in Scienze Fisiche e Astrofisiche, Indirizzo Fisica della Materia. Tirocinante presso il Dipartimento di Fisica e Astrofisica, Università degli Studi di Firenze, Firenze

\begin{abstract}
New techniques of transgenesis and imaging: applications in Neurology and Nephrology. Part I
Abstract. The emerging techniques and applications related to transgenic animals, together with the large progresses in the development of imaging systems finalized at ensuring a high-level resolution, allow nowadays to analyze the finer details of a wide range of pathophysiological phenomena. The combination of the new methods applicable in the two fields, for instance, has made possible not only to explore biological processes in vivo as they occur, but also to analyze them at a whole organ level. At the same time, it is possible to create reconstructions in two or three dimensions of the entire organ, or of its specific functional units. A fourth temporal dimension can also be added thanks to the analysis in time-lapse or to the acquisition of data in continuous. The purpose of this first of two contributions is to review the latest innovations in the fields of microscopy and transgenesis, with particular attention to how these innovations have been implemented, and which benefits derive from their evolution. The possible applications and advantages of using these systems will further be analyzed and evaluated in more detail in the next issue of this survey.
\end{abstract}

Key words: Transgenesis, Cell-population analysis, Regenerative process analysis, Single-cell resolution, Fluorescence, Imaging techniques, Resolution, Whole organ reconstruction

Conflict of interest: None.

Financial support: None.

Accettato: 26 Maggio 2014

\section{Introduzione}

Negli ultimi anni, aspirando a sempre maggiori accuratezza e veridicità nei risultati di uno studio, la ricerca di natura biomedica si è fortemente spostata dallo studio dei processi biologici in piastra a quelli in vivo. Lo studio sull'animale da laboratorio permette infatti di indagare sul comportamento di molecole, cellule, tessuti e organi in un contesto totalmente fisiologico, condizione che manca negli studi effettuati in vitro (basti pensare alla segnalazione ormonale $o$ alla regolazione del ritmo circadiano). Inoltre, tali modelli in vivo si basano sempre più su animali geneticamente modificati, al fine di permettere di identificare la singola cellula nel suo contesto fisiologico (1), di vederla cambiare di colore in base alla fase del ciclo cellulare in cui si trova (2) o di vedere come si sposta in condizioni omeostatiche così come in situazioni patologiche $(3,4)$. Da questi pochi esempi è quindi facile intuire come l'impiego di animali geneticamente modificati, o transgenici, stia cambiando drasticamente la visione che abbiamo di molti processi biologici.

\section{Gli animali transgenici}

Quando si parla di animale transgenico si parla di un animale cui sono modificati, rimossi o aggiunti uno o più determinati geni. Le finalità di queste modifiche al genoma dell'animale sono tra le più varie: è possibile studiare cosa comporti la perdita di un dato gene e che ruolo questo gene giochi nello sviluppo di un organo, oppure si possono usare geni codificanti proteine fluorescenti così da marcare popolazioni cellulari di interesse (5).

Ciò che è maggiormente importante è che l'animale porterà il transgene, stabilmente integrato nel proprio DNA, in tutte le cellule del suo corpo. Ovviamente, tra le cellule in cui troviamo il transgene, vi sono anche quelle germinali, caratteristica questa che permette la trasmissione del transgene alle future generazioni secondo una logica mendeliana. Per far sì che tutte le cellule dell'organismo possiedano le modifiche desiderate al DNA, l'ingegnerizzazione deve avvenire quando l'animale si trova ancora allo stadio di embrione: tutte le cellule che comporranno l'animale derivano infatti dall'embrione nelle 
sue prime fasi di sviluppo (5).

Per permettere l'ingegnerizzazione dell'embrione con una sequenza esogena di DNA sono state messe a punto varie tecniche, tra cui, quelle che hanno riscosso maggiore successo per rese nettamente maggiori, sono la microiniezione pronucleare e l'impiego di vettori virali.

Nell'ambito della transgenesi, la microiniezione pronucleare è sicuramente la metodica maggiormente impiegata, in quanto è considerata avere un'efficienza di delivery del DNA pari al $100 \%$. La tecnica prevede che la soluzione in cui si trova il DNA esogeno sia fisicamente iniettata nel nucleo cellulare dell'embrione, che, ricevendo il DNA estrinseco, lo integrerà stabilmente all'interno del proprio genoma con un'efficienza che è, però, decisamente inferiore a quella di delivery (il 5-10\% degli animali creati avrà il genotipo di interesse) (5).

Più recentemente sono stati invece introdotti sistemi basati su vettori virali. Tali sistemi prevedono che il gene di interesse sia veicolato mediante l'impiego di virus opportunamente modificati affinché non siano patogeni, ma che, allo stesso tempo, possiedano ancora la capacità di integrarsi permanente nel DNA dell'ospite. Tra questi virus spiccano, per alta efficienza di delivery e integrazione, retrovirus e lentivirus.

La nota dolente dei sistemi basati sui vettori virali è, però, che questi possono integrarsi casualmente nel genoma: il transgene può quindi potenzialmente collocarsi all'interno di un altro gene alterandone la funzione, così come può integrarsi in regioni silenziate o pseudo-silenziate determinando bassi livelli o addirittura la perdita di espressione del transgene stesso (5).

Per superare il problema legato all'integrazione random nel genoma, sono state sviluppate nuove metodiche che permettono di scegliere con estrema specificità dove il transgene si integrerà. Tutte queste tecniche, che prendono il nome di gene targeting, si basano sulla ricombinazione omologa. Tale processo di ricombinazione permette di inserire il transgene in un locus specifico del DNA in quanto le due estremità del frammento esogeno presentano la stessa sequenza acido-nucleica delle porzioni più esterne della regione che si vuole sostituire, modificare o eliminare. Questa omologia di sequenza nelle regioni terminali permette al transgene di affiancarsi al gene di interesse e di subire una doppia ricombinazione che risulta nella sostituzione del gene originario con il transgene (Fig. 1).

Il gene targeting può quindi essere usato per alterare la sequenza di un determinato gene al fine di renderlo non funzionale (per esempio mediante eliminazione di un introne) o addirittura per rimuovere tutta la sequenza genica: si parla in questo caso di knock-out. Allo stesso modo è possibile sostituire per inserzione un gene che fisiologicamente non sarebbe attivo, ma che, ingegnerizzato, sarà costitutivamente espresso. In questo caso si parlerà di animali knock-in.

Terminati i vari processi di modifica al genoma dell'animale, gli embrioni ingegnerizzati saranno impiantati in femmine a cui è stata indotta una pseudo-gravidanza per somministrazione di ormoni, analogamente a quanto si fa per la fecondazione assistita nell'uomo.

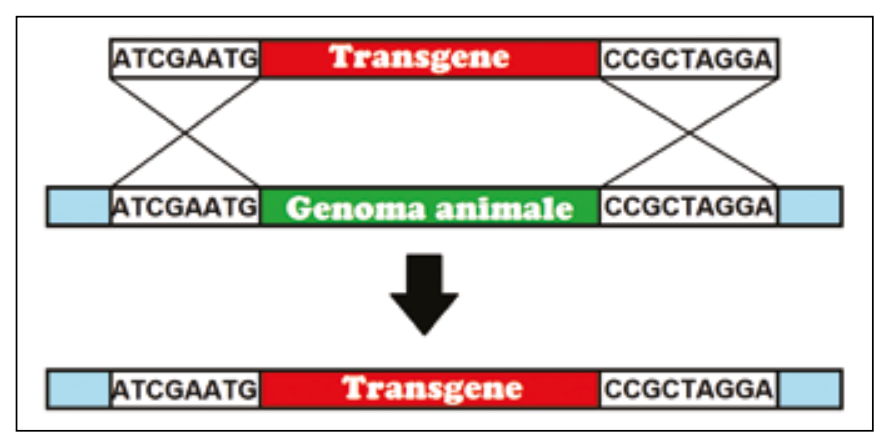

Fig. 1 - Schematizzazione del processo di ricombinazione omologa.

\section{Il lineage tracing}

Una tra le più importanti applicazioni della transgenesi è quella dell'individuazione di popolazioni cellulari nel loro contesto d'organo al fine di tracciarne il comportamento in situazioni fisiologiche o patologiche. Questo processo di individuazione e tracciamento è denominato lineage tracing e consente non solo di visualizzare, ma anche di quantificare il contributo di differenti popolazioni cellulari in diversi contesti biologici (patologici, omeostatici e rigenerativi).

Il lineage tracing si basa quindi sulla capacità di identificare una determinata cellula di interesse. Ciò è reso possibile in quanto essa esprimerà un marker (generalmente proteine fluorescenti) introdotto nel genoma sotto forma di gene reporter. Il vantaggio di avere integrato il gene reporter nel genoma dell'animale è che l'espressione della proteina fluorescente è trasferita a tutta la progenie derivante dalla cellula in analisi (6). In questo modo è perciò possibile valutare il comportamento della cellula madre e capire quanto si sia duplicata, se non lo ha fatto, che direzione abbiano preso le cellule figlie o in che processo fisiopatologico siano coinvolte.

Nel settare un'analisi di lineage tracing ciò che è, però, maggiormente importante, è la specificità con cui è possibile far esprimere proteine fluorescenti come la GFP (Green Fluorescent Protein). Infatti, sebbene solitamente l'espressione del gene reporter sia bloccata da sequenze di stop, grazie a un fine sistema di ricombinazioni genetiche indotte solo nelle cellule di interesse, è possibile rimuovere lo stop e indurre l'espressione della proteina fluorescente, così da localizzare un tipo cellulare piuttosto che un altro (Fig. 2A). La specificità di questi sistemi è determinata da promotori cellulo-specifici, ossia da molecole espresse specificamente da un dato tipo cellulare, come la Podocina nel caso dei podociti. Sfruttando la ricombinazione omologa (gene targeting), geni come la Podocina sono modificati affinché siano in grado di attivare l'espressione della proteina fluorescente e sono poi sostituiti ai geni naturalmente presenti nell'animale. Restando sul caso della Podocina, quello che succederà è che, non appena il podocita fisiologicamente attiverà l'espressione di questa proteina, assieme attiverà anche la produzione dei fattori necessari a modificare il DNA per colorare la cellula. In questo modo solo una data popolazione cellulare, in questo caso $\mathrm{i}$ podociti, acquisisce l'espressione del reporter (6).

Ciò che fanno i promotori modificati è di portare, direttamente 


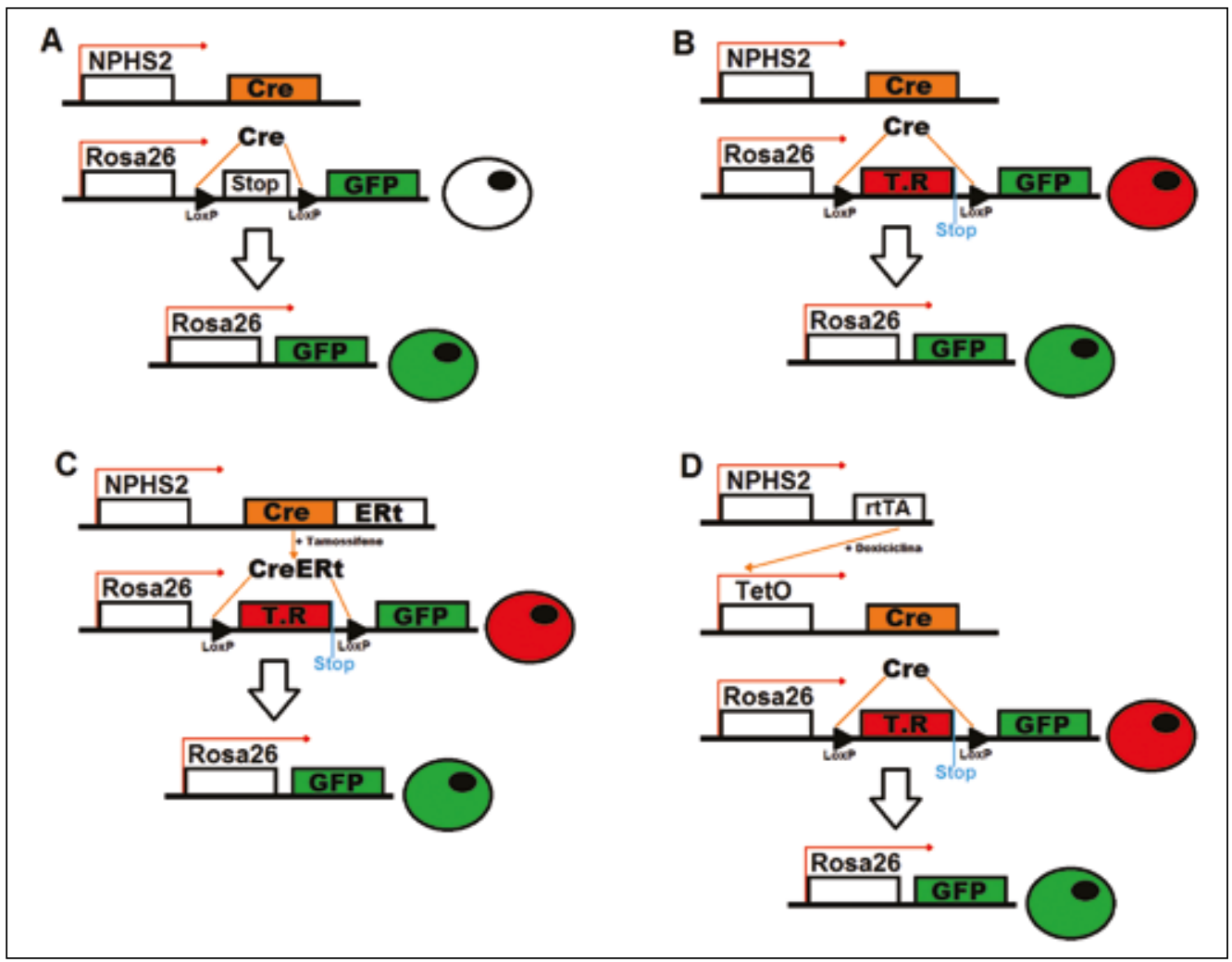

Fig. 2 - Sistemi reporter e differenti metodologie di induzione. A) Reporter verde (GFP) governato dal gene per la podocina (NPHS2). La Cre ricombinasi rimuove la cassetta di stop e ricongiunge il DNA permettendo l'espressione del verde. B) Sistema $m T / m G$ (membraneTomato/ membraneGreen) che permette la rimozione del rosso e lo switch a verde nei soli podociti. C) Sistema inducibile $\mathrm{CreER}^{\mathrm{T} 2}$ con il recettore per gli estrogeni fuso alla Cre ricombinasi, su cui esercita il controllo. D) Sistema inducibile basato sulla somministrazione di Doxiciclina. Questo sistema in particolare prevede la presenza di tre differenti transgeni, rendendo difficoltosa la gestione dei modelli. Si richiede pertanto la tripla omozigosi, così da essere sicuri che tutta la progenie abbia tutti e tre i transgeni.

o indirettamente, all'espressione dell'enzima Cre ricombinasi (Causes recombination of the bacteriophage P1 genome), attore chiave nel processo di accensione della cellula. Questo enzima media infatti la ricombinazione nel DNA che porta all'espressione della proteina fluorescente. Nello specifico, la Cre ricombinasi riconosce una sequenza nucleotidica che prende il nome di LoxP (Locus of Crossover in P1), naturalmente non presente nel genoma murino, ma introdotta nel costrutto del gene reporter. Qualora la Cre ricombinasi riconosca due siti LoxP con lo stesso orientamento, la sequenza di DNA interposta tra i due LoxP sarà rimossa e il restante DNA sarà ricongiunto (Fig. 2) (6).

Se tra i due siti LoxP è presente una sequenza di stop, a valle della quale è codificata la GFP, la GFP non potrà mai essere espressa. Ma se sarà espressa la Podocina, la cellula esprimerà anche la Cre ricombinasi che, rimuovendo la sequenza di stop interposta tra $\mathrm{i}$ due LoxP, farà accendere di verde il solo podocita (Fig. 2A).

Nel lineage tracing, il sistema reporter $m T / m G$ (membraneTomato/membraneGreen) è largamente utilizzato in quanto permette di distinguere cellule che non vanno incontro a eventi ricombinativi. Non essendo presente alcuna sequenza di stop prima del reporter rosso, tutte le cellule dell'animale esprimeranno continuamente la proteina fluorescente Tomato Red. Ma, qualora la cellula esprima la Cre ricombinasi, sarà rimossa la sequenza Tomato Red, essendo interposta tra due siti LoxP, e sarà possibile per la cellula esprimere la GFP posta a valle. In questo quadro avremo, per esempio, tutte le cellule rosse, compresi i capillari glomerulari, la capsula di Bowman e il sistema tubulare, mentre il tuft glomerulare sarà verde (Fig. 2B).

Un aspetto importante da considerare prima di scegliere l'appropriato reporter è il metodo di rilevazione. Il gene reporter scelto deve infatti possedere un sufficiente rapporto tra segnale e rumore, per evitare una rilevazione aspecifica. È comunque da tenere presente che l'impiego di molecole fluorescenti fornisce soluzioni di gran lunga superiori ai metodi basati sugli anticorpi. Esse permettono infatti di aumentare notevolmente la capacità di acquisire immagini con risoluzioni decisamente maggiori. Ciò a sua volta rende possibili ricostruzioni 3D estremamente dettagliate e ha fornito lo strumento per individuare e seguire nel processo rigenerativo varie popolazioni staminali $(4,7,8)$. Ovviamente, questa è solo la punta di un iceberg costituito da vantaggi finalizzati a una comprensione più ampia su molti sistemi in condizioni sia fisiologiche che patologiche.

I sistemi mostrati finora prevedono però l'espressione costitutiva della Cre ricombinasi: non appena il gene è espresso la cellula si colorerà. Questo può avere importanti conseguenze soprattutto in seguito allo sviluppo embrionale: se un dato organo deriva da una struttura positiva al promotore scelto, allora tutto l'organo risulta colorato da quel momento in poi. Di conseguenza non sarà più possibile effettuare un'analisi di lineage tracing all'interno di quell'organo. Per questo motivo, e per la possibilità di scegliere la finestra temporale in cui accendere le cellule positive al promotore (o marcatore), sono stati sviluppati i sistemi inducibili.

Il controllo dell'attivazione dei sistemi inducibili, o condizio- 
nali, è attuato a livello dell'espressione della Cre ricombinasi ed è regolato da un promotore modificato. Tali promotori presentano delle sequenze regolatorie che sono attivabili solo mediante somministrazione di molecole esogene. Quindi, solo in seguito alla somministrazione di tali sostanze, e solo nella cellule che esprimono il promotore, la Cre ricombinasi potrà essere espressa e potrà agire sui siti LoxP (Fig. 2C, 2D).

Grazie ai sistemi inducibili, mediante somministrazione di Tamossifene o Doxiciclina, è perciò possibile indurre l'attivazione del reporter in qualunque momento della vita dell'animale, solo da quel momento e per la durata temporale desiderata. Il più grande vantaggio che ciò comporta è che, una volta rimossa la molecola induttrice, nessuna nuova cellula che non si sia colorata nella finestra di somministrazione, sarà capace di cambiare di colore.

È così possibile marcare fisiologicamente popolazioni staminali, terminare la somministrazione dell'induttore e indurre una patologia. In condizioni patologiche potrebbe però accadere che anche differenti popolazioni cellulari esprimano il marcatore fisiologicamente associato al solo pool staminale. Se queste cellule potessero ancora ricombinare, la popolazione staminale sarebbe confusa e persa, ma siccome l'induttore non è più presente, la componente "inquinante" non si colorerà analogamente a quella staminale.

Da un punto di vista genetico, i sistemi indcibili sono ottenuti principalmente mediante due strategie. Si può infatti impiegare una Cre ricombinasi fusa con una forma modificata del recettore per gli estrogeni (CreER) e attivata dalla somministrazione di Tamossifene (Fig. 2C). Altra possibilità è quella di porre l'espressione della Cre ricombinasi sotto il controllo dell'elemento rtTA (reverse tetracycline transactivator): la Cre sarà espressa nelle cellule promotore-positive solo in presenza dell'antibiotico Doxiciclina (Fig. 2D) (9).

Al fine di studiare con maggiore accuratezza la fisiopatologia d'organo, sono stati inoltre sviluppati nuovi sistemi reporter che forniscono alle analisi di lineage tracing ulteriori vantaggi. Il transgene reporter Rainbow, per esempio, portando ogni cellula di nostro interesse ad assumere uno tra quattro colori in maniera del tutto casuale, fornisce lo strumento ideale per individuare e seguire la singola cellula marcata in omeostasi, così come in condizioni patologiche o rigenerative (10-12).

Tale sistema reporter, contrariamente a costrutti che presentano siti LoxP nello stesso orientamento, prevede anche la presenza di siti LoxP posti in maniera speculare per sequenza acido-nucleica. Ciò fornisce il vantaggio che, mentre le regioni che saranno legate dalla Cre ricombinasi riconoscendo due LoxP con lo stesso orientamento saranno eliminate (Fig. 3B), quelle legate tra due LoxP speculari saranno tagliate e invertite (Fig. 3A, 3C). Combinando queste differenti possibilità di taglio all'uso di più reporter fluorescenti posti in tandem (rosso, verde, giallo e blu) è possibile avere diverse possibilità di taglio, mutualmente esclusive, che portano l'enzima a scegliere casualmente quale colore avrà la cellula.

Con tale mezzo è quindi possibile valutare la presenza di eventuali espansioni clonali, definibili come stringhe di cellule adiacenti di uno stesso colore e perciò derivanti da una singola cellula madre. L'espansione clonale è indice del fatto che una determinata cellula, piuttosto che un'altra marcata da

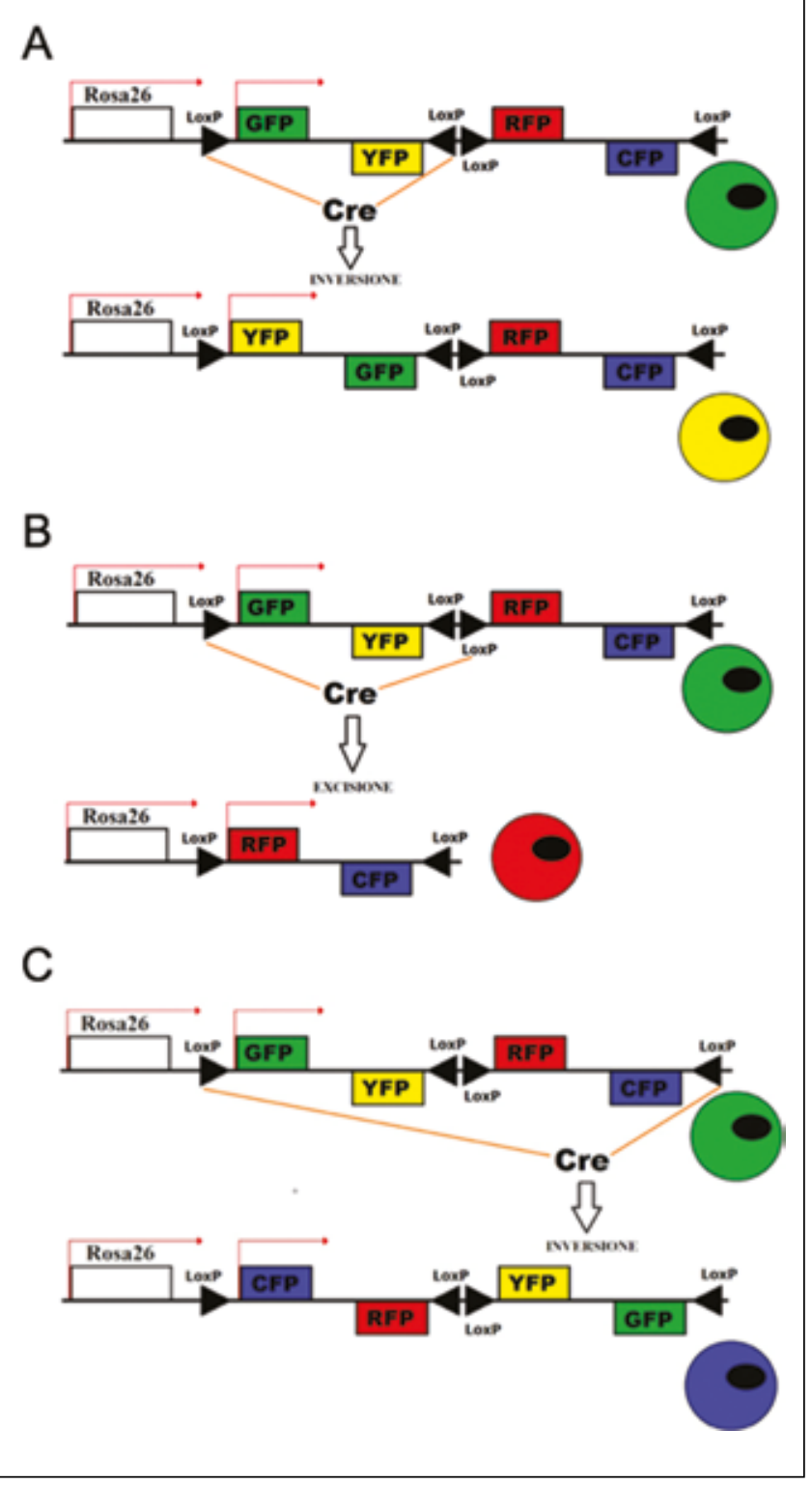

Fig. 3 - Schema sintetico delle diverse possibilità ricombinative del costrutto Rainbow.

un differente colore, si è duplicata maggiormente. Il sistema Rainbow si rivela perciò particolarmente utile nello studio delle dinamiche di popolazioni cellulari, risultando di estremo rilievo nella valutazione di compartimenti staminali in differenti organi. Il suo impiego ha permesso di analizzare i sottocompartimenti staminali presenti nelle nicchie di vari tessuti, di osservare come i differenti stadi differenziativi della cellula possano essere legati a un diverso posizionamento nella nicchia staminale e come la singola cellula staminale si comporti all'interno della nicchia stessa, in condizioni di omeostasi, così come in risposta a stimoli dannosi $(11,12)$.

Tutti i vantaggi legati alla transgenesi e alle sue più varie applicazioni richiedono però, come background, di avere efficienti sistemi che consentano di acquisire l'immagine al fine 
di poterla visualizzare e analizzare. Le tecniche di microscopia giocano quindi, nello studio di processi biologici nella loro dinamicità e struttura, un ruolo di primo piano. Esse possono infatti essere impiegate nella ricostruzione di strutture complesse come interi organi (sia espiantati che in vivo) o nella valutazione di fenomeni biologici su grande scala: ne sono un esempio la ricostruzione integrale in $3 \mathrm{D}$ del cervello o il monitoraggio dell'attività elettrica di cardiomiociti (13-16).

\section{Concetti di microscopia}

La microscopia si basa sulla rivelazione di radiazioni provenienti dai tessuti sotto esame che, nel caso della fluorescenza, sono stimolati con della radiazione a lunghezze d'onda determinate dallo spettro di assorbimento del tessuto stesso. Le molecole, dette fluorescenti, rispondono emettendo a loro volta radiazione a una lunghezza d'onda diversa da quella di eccitazione (17).

Uno dei più importanti parametri di una tecnica microscopica è la risoluzione, definita come la minima distanza per la quale due punti distinti nel campione possono essere risolti come tali. In prima approssimazione la risoluzione è direttamente proporzionale alla lunghezza d'onda $\lambda$ della radiazione rilevata e inversamente proporzionale all'apertura numerica dell'obiettivo, parametro che cresce all'aumentare dell'angolo $\Omega$ con cui l'obiettivo raccoglie la luce dal campione (Fig. 4). Inoltre anche il tempo di raccolta della luce è un parametro che può limitare la risoluzione, qualora l'efficienza di emissione delle molecole non sia sufficiente $(18-20)$.

Una spiegazione approfondita delle tecniche di microscopia va al di là dello scopo di questo articolo. Per una più facile comprensione, quando possibile, faremo riferimento come esempio alla microscopia in fluorescenza.

Con questo in mente vedremo più nel dettaglio quali sono le strutture indagabili e cosa si può ottenere attraverso le tecniche microscopiche già collaudate nel campo della biologia.

Uno dei problemi più grossi che si incontra quando si vanno a confrontare strutture la cui composizione è eterogenea, come due organi diversi o specifiche componenti all'interno di una cellula, è la perdita di informazione dovuta alla propagazione della luce in un mezzo (21). I raggi luminosi derivanti dalla fluorescenza emessa, attraversando strutture con composizioni differenti o altamente diffusive, possono essere deviati o, nel peggiore dei casi, bloccati (Fig. 5). In pratica è come quando, ponendo una sorgente luminosa dietro un foglio di carta, l'immagine della sorgente sul foglio è diversa dalla sorgente: questo è il fenomeno della diffusione (19).

Si può quindi intuire la necessità di tecniche diverse a seconda del tessuto oggetto di studio e della condizione nel quale lo si vuole osservare. Nel caso del tessuto cerebrale, per esempio, si rendono necessarie tecniche di clearing che riducono la diffusione aumentando la penetrazione della luce nel tessuto (22). Se invece si vuole studiare la trasmissione degli impulsi nervosi nell'intero cervello si possono impiegare embrioni di zebrafish, in cui il tessuto che avvolge il cervello, essendo trasparente, non ostacola né la penetrazione della luce di eccitazione né la fluorescenza in rivelazione (13).

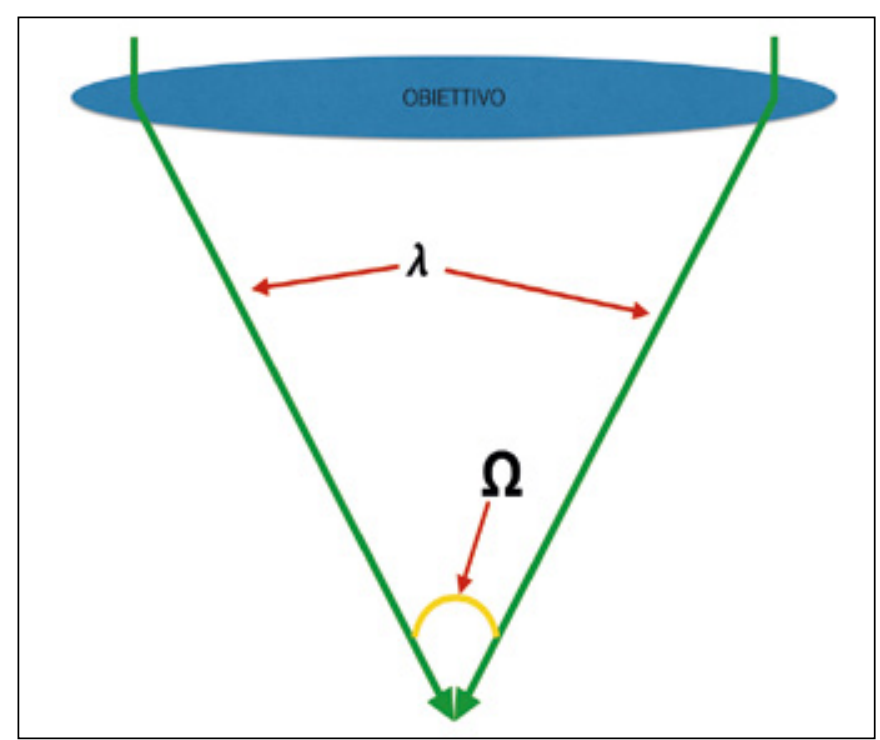

Fig. 4 - Schema di un obiettivo da microscopia, dove con $\lambda$ si è indicata la lunghezza d'onda della radiazione e con $\Omega$ l'angolo di raccolta dell'obiettivo.

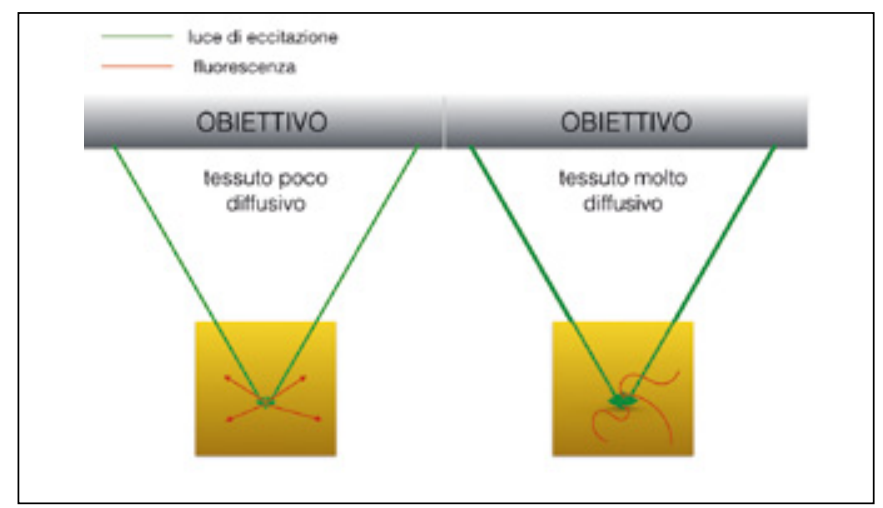

Fig. 5 - Processo di diffusione della fluorescenza (in rosso), all'interno di un tessuto molto diffusivo (destra) o poco diffusivo (sinistra).

\section{Tecniche imaging}

Seguendo un approccio in scala di lunghezze si può fare una descrizione di alcune delle tecniche di imaging più utilizzate e capire quali di queste possono essere impiegate per studiare particolari tipi di strutture biologiche (Fig. 6).

Una delle tecniche più utilizzate nell'indagine non invasiva degli organi è la DTI (Diffusion Tensor Imaging) (23), che sfrutta il principio della risonanza magnetica e che permette una risoluzione del millimetro nell'uomo e di qualche decina di micron nel topo. In questa tecnica si sfrutta la differenza di segnale emesso dalle molecole di acqua presenti in un tessuto, le quali, interagendo con un campo magnetico, si orientano di conseguenza.

Infatti le molecole di acqua presenti all'esterno delle cellule sono più mobili, mentre quelle confinate dalle pareti cellulari hanno minori possibilità di movimento. La diversa mobilità porta le molecole dell'ambiente extracellulare a una maggio- 


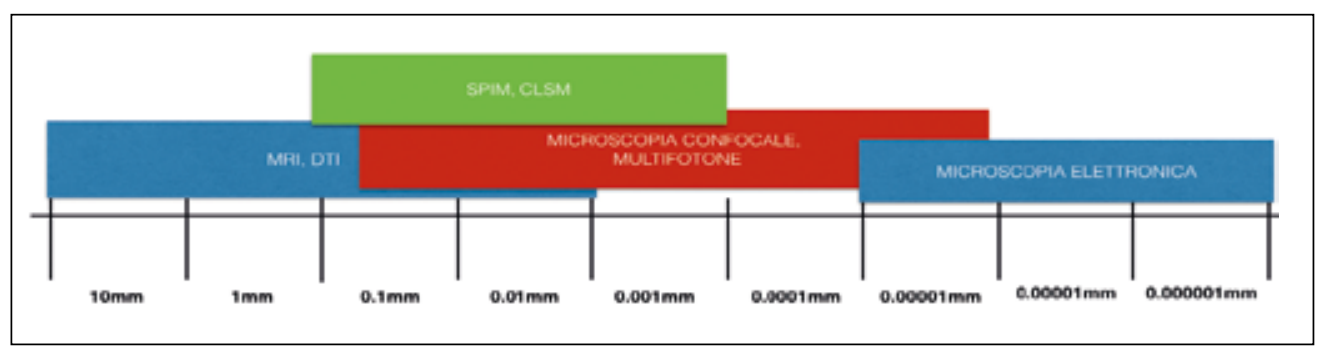

Fig. 6 - Schema per ordini di grandezza delle risoluzioni potenzialmente ottenibili con alcune delle tecniche analizzate in questo articolo.

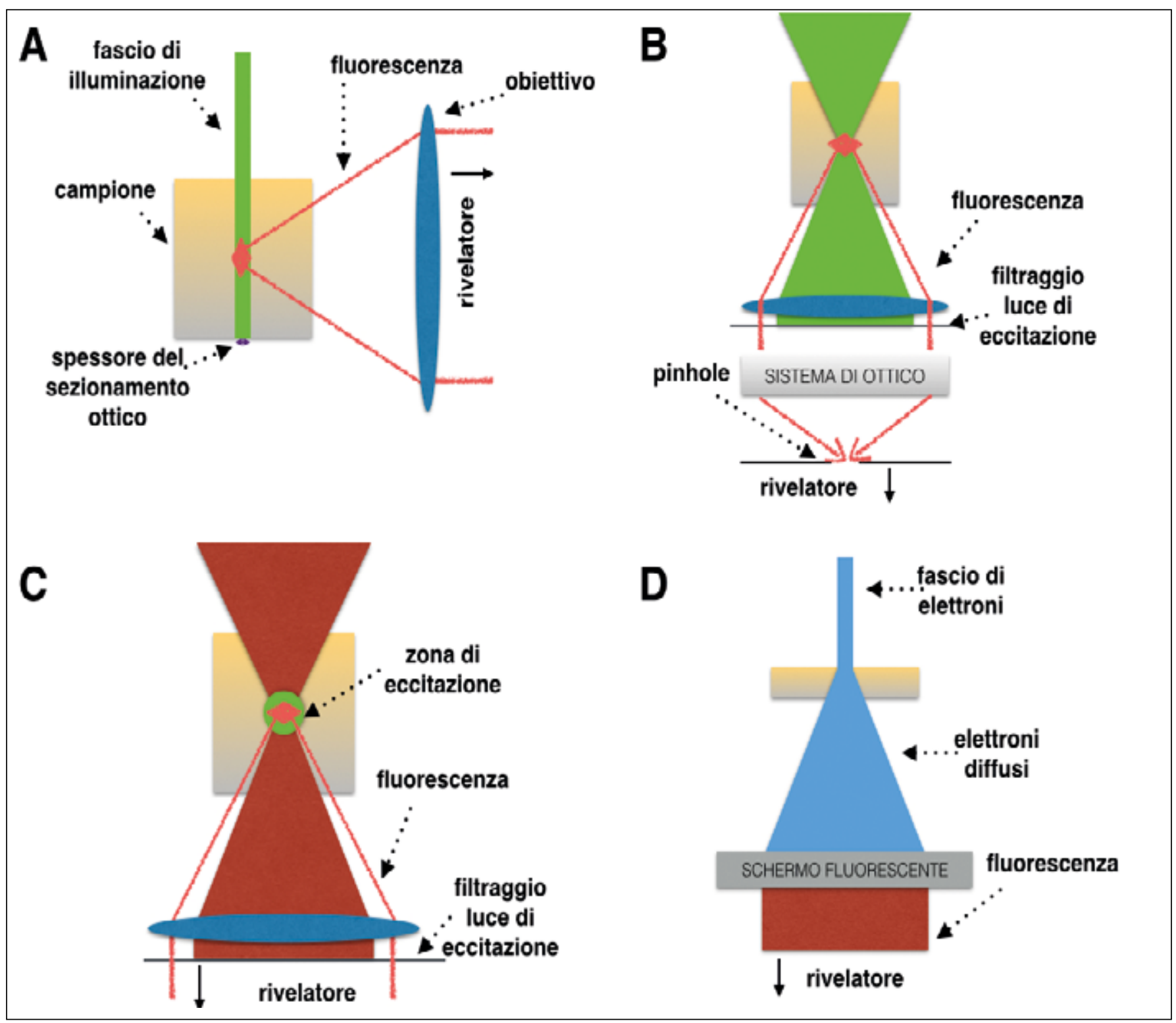

Fig. 7 - Schemi delle tecniche di microscopia, dove con il verde è indicata la regione di eccitazione e con il rosso chiaro la fluorescenza raccolta nel campo di vista dell'obiettivo. A) SPIM. B) Microscopia confocale. C) Microscopia a multifotoni. D) Microscopia elettronica in trasmissione (TEM).

re perdita di orientamento rispetto a quelle meno mobili che, conseguentemente, risalteranno maggiormente.

È così possibile acquisire immagini di tessuti con una tecnica totalmente non invasiva, grazie alla quale si possono studiare tutti i tessuti dell'organismo.
Per lo studio più approfondito di strutture come le cellule, si deve almeno riuscire a ottenere una risoluzione del micron. Le tecnologie utilizzabili su questa scala sono innumerevoli. Una delle tecniche più impiegate è la SPIM (Selective Plane Illumination Microscopy) (Fig. 7A) (24). Questa tecnica per- 
mette uno studio approfondito della struttura di interi organi come il cervello o il cuore, la ricostruzione di interi embrioni, o di interi apparati vascolari con una risoluzione potenziale di centinaia di nanometri $(14,25,26)$.

La caratteristica più importante di questa tecnica risiede nel sezionamento ottico del campione; ciò è reso possibile dal confinamento dell'illuminazione alla sola porzione del campione da cui si sta raccogliendo la luce di fluorescenza in quel momento. Si riducono così gli effetti indesiderati dati dall'eccessiva illuminazione,come il photobleaching (che porta a una riduzione dell'efficienza della fluorescenza) o la luce diffusa, che peggiorano la qualità dell'immagine (27).

Tramite questa tecnica si può, per esempio, ricostruire l'intera anatomia del cervello di un topo senza ricorrere al sezionamento fisico, il quale comporta artefatti dovuti alla non perfetta ri-sovrapposizione delle immagini passando da una sezione a un'altra.

Questa tecnica di microscopia, per la sua velocità e per il confinamento dell'illuminazione, è applicabile inoltre al monitoraggio nel lungo periodo (fino a ore) dei flussi di $\mathrm{Ca}^{2+}$ neuronali in zebrafish (13). La SPIM rende così possibile lo studio e la caratterizzazione delle correlazioni fra popolazioni di neuroni spazialmente distanti, registrando l'attività anche della singola cellula.

Altre due tecniche molto efficaci e usate nell'imaging di tessuti sono la microscopia confocale (28) e a multifotone (29), le quali riescono ad arrivare a risoluzioni dell'ordine delle centinaia di nanometri con un contrasto ottimale. La prima di queste due tecniche illumina anche una parte del campione non rilevato, ma permette di escludere in rilevazione la luce diffusa dal campione tramite un pinhole e filtri (Fig. 7B). La seconda sfrutta il confinamento dell'eccitazione alla sola zona in cui il fascio è focalizzato, aumentando di molto il rapporto fra il segnale e il rumore di fondo (Fig. 7C). Queste tecniche hanno i vantaggi di poter essere sfruttate nell'imaging in vivo e di raggiungere, potenzialmente, strutture poste fino a un millimetro di profondità nel tessuto (15).

Una tecnica che permette dei risultati eccezionali in termini di risoluzione è la microscopia elettronica $(30,31)$, la quale avrebbe teoricamente una risoluzione del picometro, ma che, nei fatti, è limitata dal rapporto segnale-rumore ai nanometri (Fig. 7D). Questa tecnica sfrutta la diffusione di elettroni nei tessuti e può essere utilizzata in trasmissione oppure in riflessione. Gli elettroni, venendo a contatto con il tessuto, deviano le loro traiettorie creando un pattern dipendente dalla struttura del campione che viene trasformato in fluorescenza da uno schermo fluorescente.

Uno degli svantaggi di questa tecnica è che necessita del sezionamento fisico con sezioni di spessore molto ridotto e non è quindi applicabile per analisi in vivo.

Oltre che allo sviluppo di nuove metodiche, la ricerca nel campo della microscopia si è concentrata sulla combinazione di una o più tra quelle precedentemente descritte. Per esempio, si sono combinate le tecniche di SPIM con quella confocale, portando alla CLSM (Confocal Light Sheet Microscopy) (14), o con quella a multifotoni: in entrambi i casi è nettamente aumentata la capacità di contrasto (32).
Dei miglioramenti nella resa si sono ottenuti, inoltre, variando le tecniche di illuminazione rispetto a quelle tradizionali, tramite l'utilizzo dell'illuminazione strutturata e con l'impiego di particolari fasci laser, detti di Bessel, molto convenienti quando si parla di tessuti diffusivi $(33,34)$.

Molte di queste tecniche di microscopia possono essere implementate con tecnologie di sezionamento. È possibile, per esempio, eseguire una scansione contemporanea al taglio e, allo stesso tempo, raccogliere la fluorescenza dalla parte ancora integra del campione. Saranno così ridotti gli effetti dovuti al sezionamento nella ricostruzione delle immagini in tre dimensioni.

Questo tipo di metodica ha non solo il limite intrinseco di non poter essere eseguita in vivo, ma richiede tempi di scansione estremamente lunghi: infatti per acquisire e ricostruire un cervello di un topo (circa $1 \mathrm{~cm}^{3}$ ) sono necessarie circa 400 ore di osservazione $(30,35)$.

\section{Conclusioni}

Lo scopo di questa trattazione è stato quello di fornire gli strumenti necessari per una prima comprensione delle più recenti metodiche impiegate nella ricerca biomedica. Partendo dalla valutazione delle tecniche di transgenesi e passando attraverso le ultime innovazioni nel campo dell'imaging, si sono analizzati alcuni tra i principali strumenti che permettono al ricercatore di indagare in maniera estremamente dettagliata molti processi biologici. Questo ha permesso di comprendere molti meccanismi inerenti le dinamiche organo-cellulari finora sconosciuti.

Da questo articolo si evince però anche l'importanza che rivestono team scientifici eterogenei: infatti, per arrivare a una comprensione globale di sistemi complessi come quelli biologici, è fondamentale l'integrazione degli input derivanti dalle varie discipline dell'area delle scienze naturali.

Se in questo articolo sono stati valutati gli aspetti tecnici di varie metodiche, le possibili applicazioni e i possibili vantaggi derivanti dall'impiego di tutti questi sistemi saranno invece oggetto di un'analisi più dettagliata nel prossimo numero di questa rubrica.

\section{Riassunto}

L'evoluzione delle tecniche e delle applicazioni legate all'impiego di animali geneticamente modificati, unite a un forte progresso nello sviluppo di sistemi di imaging volti a garantire una sempre maggiore risoluzione, permettono oggi di analizzare nei più fini dettagli un'ampia gamma di fenomeni fisiopatologici. La fusione delle nuove metodiche applicabili nei due campi ha reso possibile, per esempio, non solo l'analisi di processi biologici in vivo nel momento in cui avvengono, ma anche la loro visualizzazione a livello dell'intero organo. Allo stesso tempo è possibile creare ricostruzioni in due o in tre dimensioni dell'intero organo o di sue particolari unità funzionali. A ciò è inoltre possibile aggiungere la dimensione temporale grazie ad analisi in time lapse o per acquisizione di dati in maniera continua. La finalità di questo primo contributo è volta alla revisione 
delle più recenti innovazioni nei campi della transgenesi e della microscopia, con particolare attenzione a come tali innovazioni sono realizzate e ai vantaggi che ne derivano. Le possibili applicazioni e i vantaggi derivanti dall'impiego di tutti questi sistemi saranno invece analizzati e valutati in maggior dettaglio nel prossimo numero di questa rubrica.

Parole chiave: Transgenesi, Analisi di popolazioni cellulari, Analisi di processi rigenerativi, Risoluzione a livello della singola cellula, Fluorescenza, Tecniche di Imaging, Risoluzione, Ricostruzione di interi organi.

Dichiarazione di conflitto di interessi: Gli Autori dichiarano di non avere conflitto di interessi.
Contributi economici agli Autori: Gli Autori dichiarano di non aver ricevuto sponsorizzazioni economiche per la preparazione dell'articolo.

Indirizzo degli Autori:

Dr. Duccio Lombardi

Centro Denothe

Università degli Studi di Firenze

V.le G. Pieraccini 6

50139 Firenze

lomb.duccio@gmail.com

\section{Bibliografia}

1. Kretzschmar K, Watt FM. Lineage tracing. Cell 2012; 148 (1-2): 33-45.

2. Sakaue-Sawano A, Kurokawa H, Morimura T, et al. Visualizing spatiotemporal dynamics of multicellular cell-cycle progression. Cell 2008; 132 (3): 487-98.

3. Hackl MJ, Burford JL, Villanueva K, et al. Tracking the fate of glomerular epithelial cells in vivo using serial multiphoton imaging in new mouse models with fluorescent lineage tags. Nat Med 2013; 19 (12): 1661-6.

4. Ritsma L, Ellenbroek SI, Zomer A, et al. Intestinal crypt homeostasis revealed at single-stem-cell level by in vivo live imaging. Nature 2014; 507 (7492): 362-5.

5. Chaible LM, Corat MA, Abdelhay E, Dagli ML. Genetically modified animals for use in research and biotechnology. Genet Mol Res 2010; 9 (3): 1469-82.

6. Humphreys BD, Dirocco DP. Lineage-tracing methods and the kidney. Kidney Int 2013.

7. Barker N, van Es JH, Kuipers J, et al. Identification of stem cells in small intestine and colon by marker gene Lgr5. Nature 2007; 449 (7165): 1003-7.

8. Rios AC, Fu NY, Lindeman GJ, Visvader JE. In situ identification of bipotent stem cells in the mammary gland. Nature 2014; 506 (7488): 322-7.

9. Saunders TL. Inducible transgenic mouse models. Methods Mol Biol 2011; 693: 103-15.

10. Snippert HJ, van der Flier LG, Sato T, et al. Intestinal crypt homeostasis results from neutral competition between symmetrically dividing Lgr5 stem cells. Cell 2010; 143 (1): 134-44.

11. Ritsma L, Ellenbroek SI, Zomer A, et al. Intestinal crypt homeostasis revealed at single-stem-cell level by in vivo live imaging. Nature 2014; 507 (7492): 362-5.

12. Rios AC, Fu NY, Lindeman GJ, Visvader JE. In situ identification of bipotent stem cells in the mammary gland. Nature 2014; 506 (7488): 322-7.
13. Ahrens MB, Orger MB, Robson DN, Li JM, Keller PJ. Wholebrain functional imaging at cellular resolution using lightsheet microscopy. Nat Methods 2013; 10 (5): 413-20.

14. Silvestri L, Bria A, Sacconi L, Iannello G, Pavone FS. Confocal light sheet microscopy: micron-scale neuroanatomy of the entire mouse brain. Opt Express 2012; 20 (18): 20582-98.

15. Silvestri L, Mascaro AL, Sacconi L, Lotti J, Pavone FS. Advanced techniques to explore brain structure and function. J Innov Opt Health Sci 2013; 06: 1230002.

16. Lee $\mathrm{P}, \mathrm{K}$ los $\mathrm{M}$, Bollensdorff $\mathrm{C}$, et al. Simultaneous voltage and calcium mapping of genetically purified human induced pluripotent stem cell-derived cardiac myocyte monolayers. Circ Res 2012; 110 (12): 1556-63.

17. Lichtman JW, Conchello JA. Fluorescence microscopy. Nat Methods 2005; 2 (12): 910-9.

18. Oheim M, Beaurepaire E, Chaigneau E, Mertz J, Charpak S. Two-photon microscopy in brain tissue: parameters influencing the imaging depth. J Neurosci Methods 2001; 111 (1): 29-37.

19. Hecht E. Optics. $4^{\text {th }}$ edition. Addison Wesley, San Francisco 2002.

20. Helmchen F, Denk W. Deep tissue two-photon microscopy. Nat Methods 2005; 2 (12): 932-40.

21. Beaurepaire E, Oheim M, Mertz J. Ultra-deep two-photon fluorescence excitation in turbid media. Optics Communications 2001; 188 (1-4): 25-9.

22. Becker K, Jährling N, Saghafi S, Weiler R, Dodt HU. Chemical clearing and dehydration of GFP expressing mouse brains. PLoS One 2012; 7 (3): e33916.

23. Alexander AL, Lee JE, Lazar M, Field AS. Diffusion tensor imaging of the brain. Neurotherapeutics 2007; 4 (3): 31629.

24. Huisken J, Stainier DY. Selective plane illumination microscopy techniques in developmental biology. Development 2009; 136 (12): 1963-75.

25. Becker K, Jährling N, Kramer ER, Schnorrer F, Dodt HU. 
Ultramicroscopy: 3D reconstruction of large microscopical specimens. J Biophotonics 2008; 1 (1): 36-42.

26. Keller PJ, Schmidt AD, Wittbrodt J, Stelzer EH. Reconstruction of zebrafish early embryonic development by scanned light sheet microscopy. Science 2008; 322 (5904): 1065-9.

27. Patterson GH, Piston DW. Photobleaching in Two-Photon Excitation Microscopy. Biophys J 2000; 78 (4): 2159-62.

28. Pawley JB. HandBook of biology confocal microscopy. $3^{\text {th }}$ Edition, Springer, New York 2006.

29. Zipfel WR, Williams RM, Webb WW. Nonlinear magic: multiphoton microscopy in the biosciences. Nat Biotechnol 2003; 21 (11): 1369-77.

30. Denk W, Horstmann H. Serial block-face scanning electron microscopy to reconstruct three-dimensional tissue nanostructure. PLoS Biol 2004; 2 (11): e329.
31. Starborg T, Kalson NS, Lu Y, et al. Using transmission electron microscopy and 3 View to determine collagen fibril size and three-dimensional organization. Nat Protoc 2013; 8 (7): 1433-48.

32. Truong TV, Supatto W, Koos DS, Choi JM, Fraser SE. Deep and fast live imaging with two-photon scanned light-sheet microscopy. Nat Methods 2011; 8 (9): 757-60.

33. Mertz J. Optical sectioning microscopy with planar or structured illumination. Nat Methods 2011; 8 (10): 811-9.

34. Planchon TA, Gao L, Milkie DE, et al. Rapid three-dimensional isotropic imaging of living cells using Bessel beam plane illumination. Nat Methods 2011; 8 (5): 417-23.

35. Gong H, Zeng S, Yan C, et al. Continuously tracing brainwide long-distance axonal projections in mice at a one-micron voxel resolution. Neuroimage 2013; 74: 87-98. 